\title{
Osteosarcopenia: A Geriatric Giant of the XXI Century
}

\author{
G.Duque $e^{1,2}$

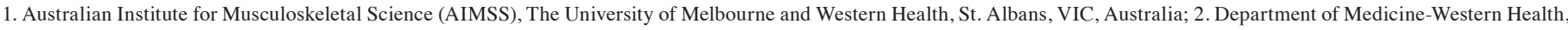 \\ The University of Melbourne, St. Albans, VIC, Australia
}

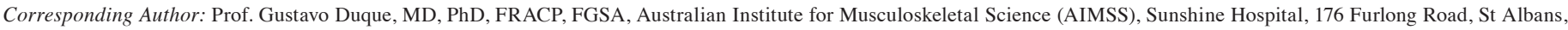
VIC, 3021, Australia, T: +61 383958121 I E: gustavo.duque@unimelb.edu.au

$\mathrm{I}$ $\mathrm{n}$ the second half of the XX century, Bernard Isaacs coined the term «geriatric giants»: immobility, instability, incontinence, and impaired intellect/memory (1). He considered them as «giants» because of «their high statistical frequency in older persons and the huge personal burden of sufferers, escalating the need for socio-medical interventions.» Since then, lots of research has been dedicated to understanding and treat those giants, especially dementia (impaired intellect/memory), where the development of a biomarker or an effective treatment has remained elusive. Nevertheless, geriatricians have adopted these giants as the main targets in their clinical practice. However, other diseases that are equally highly prevalent in older persons affecting their functional independence, and reducing their quality of life (QoL) and survival have not received the same recognition and priority in geriatrics practice. This is the case of osteoporosis and sarcopenia, which are not being widely integrated in medical training programs and clinical practice despite being characterized in the second half of the XX century. Even with a large amount of evidence, clear diagnostic criteria, and high effectiveness of current treatments, osteoporosis remains underdiagnosed and undertreated (2). On the other hand, sarcopenia was introduced in 1988 (3), and a new ICD-10-CM (M62.84) code in September 2016 represented a significant step forward in recognizing sarcopenia as a disease (4). However, and despite its clinical significance, as with osteoporosis, integrating sarcopenia in clinical practice remains challenging (5).

In 2009, in their pivotal editorial, Binkley and Buehring proposed the term «sarco-osteopenia» to describe a subset of frailer older individuals with both osteopenia/osteoporosis and sarcopenia, and that could be at a higher risk of falls, fractures, frailty and mortality (6). Since then, the term has evolved into «osteosarcopenia» and is generating growing interest from multiple angles and by several research groups worldwide (7). Together, skeletal muscle and bones constitute $55 \%$ of a healthy person's body mass, provide postural stability and facilitate movement. Interestingly, new knowledge has demonstrated that the muscle/bone interaction is significantly more complex than portrayed by traditional siloed approaches that have focused on and studied these organs separately while limiting their interaction to primarily mechanical. We now understand that, in addition to their anatomical connections, muscles and bones communicate via hormonal and biochemical messengers (8). Received April 2, 2021
Importantly, these biochemical interactions and endocrine roles affect other organs and systems; thus, alterations in bone and muscle mass and function have clinical consequences that go far beyond the musculoskeletal system (9).

Some questioners of the term osteosarcopenia have referred to the multiple cross-talks between organs and systems that could be equally affected by disease. Indeed, although «cardiosarcopenia» could be observed in heart failure, or «thyrosteoporosis» could occur in hyper or hypothyroidism, there are multiple reasons to propose osteosarcopenia as a separate entity, consequence of shared pathophysiology, with common clinical outcomes and potential combined therapeutic targets. Importantly, no two tissues in the body show a similar decline in their structure and function under aging and pathological conditions as bone and muscle do. For instance, immobility (one of the geriatric giants) is rapidly associated with a decline in both tissues, thus predisposing to adverse outcomes (i.e. falls, disability), which prevents an effective response to rehabilitation (10).

Regarding its pathophysiology, osteosarcopenia is the consequence of multi-directional abnormalities in the bone muscle cross-talk and local changes that have been fully summarized elsewhere (11), and that could be closely associated with the geroscience model (12), in which several age-related diseases share similar pathophysiological mechanisms. Interestingly, fat has become a third player in the pathophysiology of osteosarcopenia (13), not necessarily because of the presence of obesity, but because of the increasing levels of fat infiltration that are observed inside the bone marrow in osteoporotic bone and the dramatic levels of intramuscular fat infiltration observed in sarcopenic muscle (13). These high levels of fat infiltration, which are independent of the subject body mass index, are known to be lipotoxic, affecting the function and structure of the local tissues (14). Whether high levels of fat infiltration could be used as a diagnostic or therapeutic tool for osteosarcopenia remains unknown but offers a novel and exciting approach that deserves further exploration.

A major source of confusion in the field comes from a specific clinical phenotype and adverse outcomes associated with osteosarcopenia (15). Being a geriatric syndrome (giant) and not a specific disease, osteosarcopenia is considered as the combined presence of osteopenia/osteoporosis and sarcopenia in the same patient, which is expected to predispose them to adverse outcomes such as falls, fractures, frailty, disability, 
Figure 1. Osteosarcopenia renders older people significantly more vulnerable to adverse outcomes. A single fall or fracture markedly increases the risk of future events. Fear of falling and injury then erodes physical confidence, swiftly followed by compromised mobility, independence, and overall wellbeing

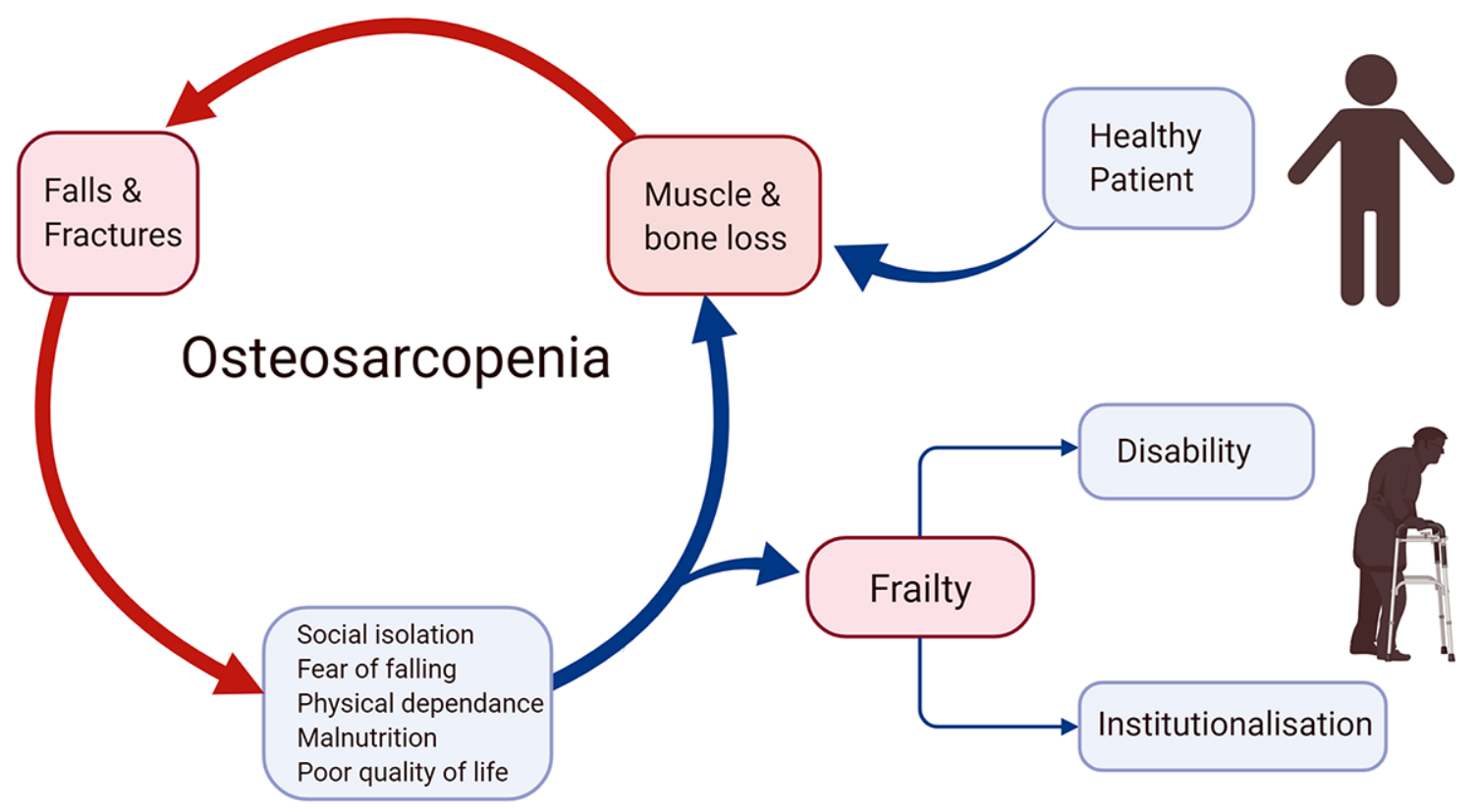

reduced QoL, and increased mortality. However, exploring this association has been challenging due to the original design of multiple clinical studies in the field, which did not include clear criteria for osteosarcopenia or used a potpourri of sarcopenia definitions $(16,17)$. Nevertheless, recent studies have attempted to investigate the existence of a specific ostesarcopenia phenotype and its association with adverse outcomes in older persons. Regarding prevalence, it ranges between 5-37\% in community-dwelling older adults, with the highest rates observed in those with fractures (7). Risk factors associated with osteosarcopenia include older age, sex [men: 14.3\% (60$64 \mathrm{yrs})$ to $59.4 \%$ ( $\geq 75 \mathrm{yrs}$ ); women: $20.3 \%$ (60-64yrs) to $48.3 \%$ ( $\geq 75 \mathrm{yrs})]$, physical inactivity, and low BMI $(7,18)$. Among 148 geriatric inpatients, osteosarcopenic individuals demonstrate poorer nutritional status versus osteoporosis or sarcopenia alone (19). Interestingly, osteosarcopenia was also highly prevalent in older subjects with high serum levels of parathyroid hormone in the absence of vitamin D deficiency or renal failure (20).

By simply defining osteosarcopenia as the combination of sarcopenia (independently of the definition used) and osteopenia/osteoporosis (defined using densitometry criteria), several teams have demonstrated that osteosarcopenic patients are at a higher risk of adverse outcomes (21-24). In 253 older subjects ( $77 \%$ women; aged $77.9 \pm 0.42$ years) assessed at our Falls and Fractures Clinic (Western Health, Melbourne), we found that osteosarcopenia was associated with worse function and balance. Additionally, the presence of severe sarcopenia in osteosarcopenic patients conferred an increased rate of falls [odds ratios (ORs) from 2.83 to 3.63 ; $\mathrm{P}<.05$ for all] and fractures (ORs from 3.86 to 4.38 ; $\mathrm{P}<.05$ for all) when employing the European Working Group on Sarcopenia in
Older People (EWGSOP2) and Foundation for the National Institutes of Health (FNIH) definitions, respectively (22). Furthermore, in a Chilean cohort of 1119 participants (68.5\% female; mean age 72 years), Salech et al. (23) reported that osteosarcopenia was a predictor of falls, fractures, and mortality in this population. High levels of mortality in osteosarcopenic subjects were also observed in the Geelong Osteoporosis Study (24). Overall, the evidence on the association between osteosarcopenia and a higher risk of adverse outcomes when compared to osteoporosis or sarcopenia alone is growing. This could induce osteosarcopenic subjects to enter into a dynamic vicious cycle that could result in frailty, disability, institutionalization, and premature death (Figure 1).

However, some authors expect this augmented risk of adverse outcomes to be the result of one plus one equal two. In the case of osteosarcopenia, this is not as simple because here we are describing a syndrome composed of two diseases sharing a common pathophysiology and risk factors and simultaneously occurring in the same subject, thus the presence of osteosarcopenia and the physiological disturbances generated by this syndrome could lead to new equations that could also have cardiometabolic implications. This intriguing hypothesis could only be tested by designing studies that specifically look at osteosarcopenia using a highly needed standardized definition for sarcopenia without excluding osteopenic subjects; a population that represents $50 \%$ of minimal trauma fractures, which could be precipitated by the simultaneous presence of sarcopenia (22).

Regarding biomarkers and treatments for osteosarcopenia, the current evidence is limited mainly to non-pharmacological interventions $(25,26)$. Nevertheless, the growing understanding 
of the pathophysiology and clinical phenotype of this syndrome has generated a variety of approaches that have involved geroscience-related strategies (12). New knowledge on the role of stem cells in the pathophysiology of this syndrome has allowed us and others to propose quantification and/or characterization of these cells (which are common precursors of muscle and bone) as a new diagnostic and therapeutic approach to osteosarcopenia $(27,28)$. Also, the associations between hormones such as sex steroids or vitamin D and osteosarcopenia have opened new potential diagnostic and therapeutic approaches to this condition $(20,29-31)$. Specifically for treatment, having a drug with a dual therapeutic effect on bone and muscle would constitute a significant advancement in geriatrics and musculoskeletal fields (32). In contrast to osteoporosis, where pharmacological treatments are very effective, pharmacological approaches for sarcopenia have been mostly disappointing $(33,34)$, possibly because these drugs are being directed to the wrong biological target. Interestingly, there is growing evidence that Denosumab, an antibody against receptor activator of nuclear factor kappa-B ligand (RANKL) widely used for osteoporosis, could be that very much searched compound with dual effect on muscle and bone. However, more evidence is still needed (35). Overall, a combined therapeutic approach that would simultaneously improve muscle condition and bone mass while reducing the risk for falls and fractures and their associated adverse outcomes is still lacking.

In summary, osteosarcopenia should be considered a geriatric giant of the XXI century due to its high prevalence in older persons and the vast personal burden of sufferers. To understand and effectively diagnose and treat osteosarcopenia, we still require a clear definition of sarcopenia, innovative biomarkers and diagnostic methods, and interventions with dual effect on bone and muscle. As a combination of two somewhat neglected and recently understood conditions, awareness of osteosarcopenia as a geriatric syndrome (36) should be increased, and its prevention, identification and treatment should become a priority in our geriatrics practice. In a set of fragmented fields in which osteoporosis and sarcopenia and falls and fracture risk are identified and managed at different settings and by various specialties and teams, osteosarcopenia could be the unifier concept that will finally improve care to these devastating conditions.

Conflict of interest: No conflict of interest to declare.

Ethical standards: The author declares that the review comply with the current laws of the country in which it was performed

\section{References}

1. Isaacs B. Ageing and the doctor. In: Hobman D, ed. The Impact of Ageing. London: Croom Helm; 1981.

2. Lombardi F, Paoletti L, Carrieri B, Dell'Aquila G, Fedecostante M, Di Muzio M, Corsonello A, Lattanzio F, Cherubini A. Underprescription of medications in older adults: causes, consequences and solutions-a narrative review. Eur Geriatr Med. 2021 Mar 11. doi: 10.1007/s41999-021-00471-x.

3. Rosenberg IH. Sarcopenia: origins and clinical relevance. J Nutr 1997;127(Suppl:990S-991S.

4. Anker SD, Morley JE, von Haehling S. Welcome to the ICD-10 code for sarcopenia. J Cachexia Sarcopenia Muscle. 2016 Dec;7(5):512-514. doi: 10.1002/jcsm.12147.

5. Bauer J, Morley JE, Schols AMWJ, Ferrucci L, Cruz-Jentoft AJ, Dent E, Baracos VE, Crawford JA, Doehner W, Heymsfield SB, Jatoi A, Kalantar-Zadeh K, Lainscak M,
Landi F, Laviano A, Mancuso M, Muscaritoli M, Prado CM, Strasser F, von Haehling S, Coats AJS, Anker SD. Sarcopenia: A Time for Action. An SCWD Position Paper. J Cachexia Sarcopenia Muscle. 2019 Oct;10(5):956-961. doi: 10.1002/jcsm.12483.

6. Binkley N, Buehring B. Beyond FRAX: it's time to consider "sarco-osteopenia". J Clin Densitom. 2009 Oct-Dec;12(4):413-6. doi: 10.1016/j.jocd.2009.06.004.

7. Kirk B, Zanker J, Duque G. Osteosarcopenia: epidemiology, diagnosis, and treatmentfacts and numbers. J Cachexia Sarcopenia Muscle. 2020 Jun;11(3):609-618. doi: $10.1002 / \mathrm{jcsm} .12567$.

8. Kirk B, Feehan J, Lombardi G, Duque G. Muscle, Bone, and Fat Crosstalk: the Biological Role of Myokines, Osteokines, and Adipokines. Curr Osteoporos Rep. 2020 Aug;18(4):388-400. doi: 10.1007/s11914-020-00599-y.

9. Bosco F, Musolino V, Gliozzi M, Nucera S, Carresi C, Zito MC, Scarano F, Scicchitano M, Reale F, Ruga S, Maiuolo J, Macrì R, Guarnieri L, Coppoletta AR, Mollace R, Muscoli C, Palma E, Mollace V. The muscle to bone axis (and viceversa): An encrypted language affecting tissues and organs and yet to be codified? Pharmacol Res. 2021 Mar;165:105427. doi: 10.1016/j.phrs.2021.105427.

10. Compston J. Emerging therapeutic concepts for muscle and bone preservation building. Bone. 2015 Nov;80:150-156. doi: 10.1016/j.bone.2015.04.013.

11. He C, He W, Hou J, Chen K, Huang M, Yang M, Luo X, Li C. Bone and Muscle Crosstalk in Aging. Front Cell Dev Biol. 2020 Dec 10;8:585644. doi: 10.3389/ fcell.2020.585644.

12. Kirk B, Al Saedi A, Duque G. Osteosarcopenia: A case of geroscience. Aging Med (Milton). 2019 Sep 8;2(3):147-156. doi: 10.1002/agm2.12080.

13. Hirschfeld HP, Kinsella R, Duque G. Osteosarcopenia: where bone, muscle, and fat collide. Osteoporos Int. 2017 Oct;28(10):2781-2790. doi: 10.1007/s00198-017-4151-8.

14. Al Saedi, A, Goodman C,, Myers D, Hayes A, Duque G (2019) Osteosarcopenia as a Lipotoxic Disease. In: Osteosarcopenia: Bone, Muscle and Fat Interactions. Duque, Gustavo, ed. Springer Nature, Cham, USA, pp. 123-143.

15. Zanker J, Duque G. Osteosarcopenia: the Path Beyond Controversy. Curr Osteoporos Rep. 2020 Apr;18(2):81-84. doi: 10.1007/s11914-020-00567-6.

16. Scott D, Seibel M, Cumming R, Naganathan V, Blyth F, Le Couteur DG, Handelsman DJ, Waite LM, Hirani V. Does Combined Osteopenia/Osteoporosis and Sarcopenia Confer Greater Risk of Falls and Fracture Than Either Condition Alone in Older Men? The Concord Health and Ageing in Men Project. J Gerontol A Biol Sci Med Sci. 2019 May 16;74(6):827-834. doi: 10.1093/gerona/gly 162.

17. Balogun S, Winzenberg T, Wills K, Scott D, Callisaya M, Cicuttini F, Jones G, Aitken D. Prospective associations of osteosarcopenia and osteodynapenia with incident fracture and mortality over 10 years in community-dwelling older adults. Arch Gerontol Geriatr. 2019 May-Jun;82:67-73. doi: 10.1016/j.archger.2019.01.015.

18. Huo YR, Suriyaarachchi P, Gomez F, Curcio CL, Boersma D, Muir SW, MonteroOdasso M, Gunawardene P, Demontiero O, Duque G. Phenotype of osteosarcopenia in older individuals with a history of falling. J Am Med Dir Assoc. 2015 Apr;16(4):290-5. doi: 10.1016/j.jamda.2014.10.018.

19. Fahimfar N, Zahedi Tajrishi F, Gharibzadeh S, Shafiee G, Tanha K, Heshmat R et al Prevalence of Osteosarcopenia and Its Association with Cardiovascular Risk Factors in Iranian Older People: Bushehr Elderly Health (BEH) Program. Calcif Tissue Int 2019 doi:10.1007/s00223-019-00646-6.

20. Suriyaarachchi P, Gomez F, Curcio CL, Boersma D, Murthy L, Grill V, Duque G. High parathyroid hormone levels are associated with osteosarcopenia in older individuals with a history of falling. Maturitas. 2018 Jul;113:21-25. doi: 10.1016/j maturitas.2018.04.006.

21. Reiss J, Iglseder B, Alzner R, Mayr-Pirker B, Pirich C, Kässmann H et al. Sarcopenia and osteoporosis are interrelated in geriatric inpatients. Z Gerontol Geriatr 2019 doi:10.1007/s00391-019-01553-z.

22. Sepúlveda-Loyola W, Phu S, Bani Hassan E, Brennan-Olsen SL, Zanker J, Vogrin S, Conzade R, Kirk B, Al Saedi A, Probst V, Duque G. The Joint Occurrence of Osteoporosis and Sarcopenia (Osteosarcopenia): Definitions and Characteristics. J Am Med Dir Assoc. 2020 Feb;21(2):220-225. doi: 10.1016/j.jamda.2019.09.005.

23. Salech F, Marquez C, Lera L, Angel B, Saguez R, Albala C. Osteosarcopenia Predicts Falls, Fractures, and Mortality in Chilean Community-Dwelling Older Adults. J Am Med Dir Assoc. 2020 Sep 10:S1525-8610(20)30657-5. doi: 10.1016/j. jamda.2020.07.032

24. Pasco JA, Mohebbi M, Holloway KL, Brennan-Olsen SL, Hyde NK, Kotowicz MA. Musculoskeletal decline and mortality: prospective data from the Geelong Osteoporosis Study. J Cachexia Sarcopenia Muscle. 2017 Jun;8(3):482-489. doi $10.1002 / \mathrm{jcsm} .12177$

25. Atlihan R, Kirk B, Duque G. Non-Pharmacological Interventions in Osteosarcopenia: A Systematic Review. J Nutr Health Aging. 2021;25(1):25-32. doi: 10.1007/s12603 020-1537-7.

26. Ghasemikaram M, Chaudry O, Nagel AM, Uder M, Jakob F, Kemmler W, Kohl M, Engelke K. Effects of 16 months of high intensity resistance training on thigh muscle fat infiltration in elderly men with osteosarcopenia. Geroscience. 2021 Jan 15. doi: 10.1007/s11357-020-00316-8.

27. Feehan J, Kassem M, Pignolo RJ, Duque G. Bone From Blood: Characteristics and Clinical Implications of Circulating Osteogenic Progenitor (COP) Cells. J Bone Miner Res. 2021 Jan;36(1):12-23. doi: 10.1002/jbmr.4204. Epub 2020 Nov 12.

28. Gimble J. Mesenchymal Stem Cells as Regulators of Bone, Muscle, and Fat 
Formation. In: Osteosarcopenia: Bone, Muscle and Fat Interactions. Duque, Gustavo, ed. Springer Nature, Cham, USA, pp. 123-143.

29. Laurent MR, Dedeyne L, Dupont J, Mellaerts B, Dejaeger M, Gielen E. Age-related bone loss and sarcopenia in men. Maturitas. 2019 Apr;122:51-56. doi: 10.1016/j. maturitas.2019.01.006.

30. Kirk B, Miller S, Zanker J, Duque G. A clinical guide to the pathophysiology, diagnosis and treatment of osteosarcopenia. Maturitas. 2020 Oct;140:27-33. doi 10.1016/j.maturitas.2020.05.012.

31. Bruyère $\mathrm{O}$, Cavalier $\mathrm{E}$, Reginster JY. Vitamin $\mathrm{D}$ and osteosarcopenia: an update from epidemiological studies. Curr Opin Clin Nutr Metab Care. 2017 Nov;20(6):498-503. doi: 10.1097/MCO.0000000000000411.

32. Girgis CM, Mokbel N, Digirolamo DJ. Therapies for musculoskeletal disease: can we treat two birds with one stone? Curr Osteoporos Rep. 2014 Jun;12(2):142-53. doi: 10.1007/s11914-014-0204-5.

33. Becker C, Lord SR, Studenski SA, Warden SJ, Fielding RA, Recknor CP, Hochberg MC, Ferrari SL, Blain H, Binder EF, Rolland Y, Poiraudeau S, Benson CT, Myers SL, Hu L, Ahmad QI, Pacuch KR, Gomez EV, Benichou O; STEADY Group. Myostatin antibody (LY2495655) in older weak fallers: a proof-of-concept, randomised, phase 2 trial. Lancet Diabetes Endocrinol. 2015 Dec;3(12):948-57. doi: 10.1016/S2213 8587(15)00298-3.
34. Rooks D, Swan T, Goswami B, Filosa LA, Bunte O, Panchaud N, Coleman LA, Miller RR, Garcia Garayoa E, Praestgaard J, Perry RG, Recknor C, Fogarty CM, Arai H, Chen LK, Hashimoto J, Chung YS, Vissing J, Laurent D, Petricoul O, Hemsley S, Lach-Trifilieff E, Papanicolaou DA, Roubenoff R. Bimagrumab vs Optimized Standard of Care for Treatment of Sarcopenia in Community-Dwelling Older Adults: A Randomized Clinical Trial. JAMA Netw Open. 2020 Oct 1;3(10):e2020836. doi: 10.1001/jamanetworkopen.2020.20836.

35. Wu XD, Huang W, Qiu GX. Denosumab for the Prevention of Falls in Older People: We Need More Evidence. J Bone Miner Res. 2020 Aug;35(8):1609-1610. doi $10.1002 / \mathrm{jbmr} .4051$

36. Hassan EB, Duque G. Osteosarcopenia: A new geriatric syndrome. Aust Fam Physician. 2017 Nov;46(11):849-853. 